\title{
Hyoid Muscle
}

National Cancer Institute

\section{Source}

National Cancer Institute. Hyoid Muscle. NCI Thesaurus. Code C32753.

One of a group of neck muscles attached to the hyoid bone used in mastication and swallowing, which are classified as either suprahyoid muscles, the stylohyoid, digastric, mylohyoid, and geniohyoid, or infrahyoid muscles, the omohyoid, sternohyoid, thyrohyoid, and sternothyroid. 